\title{
Reciprocal connectivity of the periaqueductal gray with the ponto-medullary respiratory network in rat.
}

Pedro Trevizan-Baú1, Werner I. Furuya1, Stuart B. Mazzone², Davor Stanić1, Rishi R. Dhingra ${ }^{1}$, Mathias Dutschmann ${ }^{1 *}$

${ }^{1}$ The Florey Institute of Neuroscience and Mental Health, Discovery Neuroscience theme, The University of Melbourne, Parkville, VIC, 3010, Australia ${ }^{2}$ Department of Anatomy and Neuroscience, The University of Melbourne, Parkville, VIC, 3010, Australia

\section{Correspondence:}

Mathias Dutschmann, Ph.D., The Florey Institute of Neuroscience and Mental Health, Discovery Neuroscience, Team Respiratory Neurobiology, Howard Florey Laboratories, 30 Royal Parade, Parkville, Victoria, 3052, Australia. Phone: +61 3 90356520; e-mail: mathias.dutschmann@florey.edu.au 


\section{Highlights}

- The lateral and ventrolateral PAG project to the primary respiratory network.

- The Kölliker-Fuse nucleus shares the densest reciprocal connectivity with the PAG.

- The Bötzinger complex appears to have very little connectivity with the PAG. 


\section{Abstract}

Synaptic activities of the periaqueductal gray (PAG) can modulate or appropriate the respiratory motor activities in the context of behavior and emotion via descending projections to nucleus retroambiguus. However, alternative anatomical pathways for the mediation of PAG-evoked respiratory modulation via core nuclei of the brainstem respiratory network remains only partially described. We injected the retrograde tracer Cholera toxin subunit B (CT-B) in the pontine Kölliker-Fuse nucleus (KFn, n=5), medullary Bötzinger (BötC, $n=3$ ) and pre-Bötzinger complexes (pre-BötC; $n=3$ ), and the caudal raphé nuclei $(n=3)$, and quantified the ascending and descending connectivity of the PAG. CT-B injections in the $\mathrm{KFn}$, pre-BötC, and caudal raphé, but not in the BötC, resulted in CT-B-labeled neurons that were predominantly located in the lateral and ventrolateral PAG columns. In turn, CT-B injections into the lateral and ventrolateral PAG columns $(n=4)$ yield the highest numbers of CT-B-labeled neurons in the KFn and far fewer numbers of labeled neurons in the pre-BötC and caudal raphé. Analysis of the relative projection strength revealed that the KFn shares the densest reciprocal connectivity with the PAG (ventrolateral and lateral columns, in particular). Overall, our data imply that the PAG may engage a distributed respiratory rhythm and pattern generating network beyond the nucleus retroambiguus to mediate downstream modulation of breathing. However, the reciprocal connectivity of the KFn and PAG suggests specific roles for synaptic interaction between these two nuclei that are most likely related to the regulation of upper airway patency during vocalization or other volitional orofacial behaviors.

Keywords: behavioral modulation of breathing, emotional modulation of breathing, limbic system, vocalization, orofacial behavior, inspiratory off-switch. 


\section{Introduction}

Stimulation of the lateral and ventrolateral cell columns of the midbrain periaqueductal gray (IPAG and VIPAG, respectively) can elicit behavioral modulations and appropriations of ongoing respiratory activity, in particular during vocalization (Magoun et al., 1937; Jürgens and Pratt, 1979, Larson and Kistler, 1984; Jürgens, 1994; Jürgens, 2009). In addition, these cell columns of the PAG have function in the mediation of cardio-respiratory effects associated with emotions (Carrive et al., 1997; Fanselow, 1991; Walker and Carrive, 2003). Thus, the PAG is seen as the primary interface that connects cortical and limbic forebrain circuits with autonomic networks in the brainstem (Dampney et al., 2013; Subramanian and Holstege, 2014).

The neuroanatomical framework for PAG-evoked modulation of cardio-respiratory activity is linked to PAG descending projections to the nucleus retroambiguus (NRA) in the caudal medulla oblongata (Holstege et al., 1989; Holstege et al., 1997; Vanderhorst et al., 2000). In turn, the NRA projects to motoneurons of the nucleus ambiguus (Vanderhorst et al., 2001) and to various pools of respiratory motoneurons in the ventral horn of the spinal cord (Vanderhorst and Holstege, 1995; Holstege et al., 1997). Therefore, the PAG-NRA pathway is considered essential for PAG-mediated volitional and emotional modulation of breathing (Subramanian et al., 2008a, b; Subramanian and Holstege, 2014; Faul et al., 2019). However, while the NRA has a role in respiratory rhythm generation (Jones et al., 2012), it does not contribute to the generation of the respiratory motor pattern (Jones et al., 2016a), which depends on synaptic interactions between the rhythmogenic pre-Bötzinger complex (pre-BötC; Smith et al., 1991; Feldman and Del Negro, 2006) in the rostral medulla, and pontine circuits (Jones et al., 2016b). Given that the PAG is not an integral part of the respiratory pattern generating circuit (Farmer et al., 2014), the mediation of respiratory modulation via the PAG-NRA pathway may not bypass the ponto-medullary rhythm and pattern generating network. Indeed, various anterograde and retrograde tracing studies 
have reported that descending PAG projections also target broader pontine and medullary brainstem areas, including the pontine tegmentum (Holstege, 1991), the rostral ventrolateral medulla (Carrive et al., 1988; Yasui et al., 1990), subretrofacial nucleus (Carrive et al.,1989; Carrive and Bandler, 1991), and the caudal raphé nuclei (Beitz et al., 1983; van Bockstaele et al., 1991; Cowie and Holstege, 1992). However, many of these studies were performed in cat and were often focused on general PAG pathways linked to cardiorespiratory regulation during defence behavior. The specific connectivity with core nuclei of the brainstem respiratory rhythm and pattern generating network were often not specifically investigated, except in the work of Krout et al. (1998) who reported a modest amount of anterograde-labeled fibres in the Kölliker-Fuse nucleus (KFn), a core nucleus of the pontine respiratory group (Dutschmann and Dick, 2012).

To develop a better understanding of the anatomical frameworks for the PAGmediated modulation of breathing in the context of emotion and behavior, we specifically assessed the relative strength of the reciprocal connectivity of the IPAG and vIPAG with core nuclei of the respiratory network such as the Bötzinger complex (BötC), pre-BötC, KFn, and the caudal raphé nuclei using a retrograde tracing approach. 


\section{Results}

We microinjected the retrograde tracer CT-B (150 nL) unilaterally in the pontine KFn $(n=5)$, the medullary $\operatorname{BötC}(n=3)$ and pre-BötC $(n=3)$, the caudal raphé $(n=3)$, or the midbrain PAG ( $n=4)$. CT-B injections were anatomically confined to discrete injection sites in the brainstem target areas (see representative photographs, Fig. $1 \& 3 \mathrm{~A}$ ). Note that the same tracer injections were reported in a most recent study from our laboratory that performed an extensive analysis of descending forebrain inputs to these brainstem nuclei (Trevizan-Baú et al., 2020).

\subsection{Distribution of retrogradely labeled neurons in the PAG after CT-B injections in ponto-medullary respiratory nuclei}

The numbers of CT-B-retrogradely labeled cell bodies in the ipsilateral and contralateral midbrain PAG was $448 \pm 121$ labeled neurons per case $(\mathrm{n} / \mathrm{c})$ after CT-B injection in the pontine KFn, and $303 \pm 98 \mathrm{n} / \mathrm{c}$ and $353 \pm 109 \mathrm{n} / \mathrm{c}$ after CT-B injection in the medullary preBötC and caudal raphé, respectively (Fig. 2A). However, no CT-B-labeled neurons were detected following tracer injection in the BötC (Fig. 2A). Analysis of the ipsilateral and contralateral distribution of CT-B-labeled neurons (Fig. 2A) revealed a significant ipsilateral dominance of descending PAG projections targeting the KFn (394 \pm 109 vs. $55 \pm 15$ neurons/case $(n / c) ; p<0.05)$, and a tendency of ipsilateral PAG projections to the pre-BötC ( $247 \pm 84$ vs. $56 \pm 16 \mathrm{n} / \mathrm{c}$ ). Because injections in the caudal raphé were in the midline of the medulla, we observed similar numbers of CT-B labeled neurons in the left and right hemisphere of the PAG (data not shown).

Analysis of the distribution of CT-B-labeled neurons in specific longitudinal cell columns of the PAG, which include the dorsomedial (dmPAG), dorsolateral (dIPAG), lateral (IPAG), and ventrolateral (vIPAG) columns (Bandler et al., 1991; Carrive, 1993), revealed that the majority of the projection neurons were located in the IPAG and vIPAG (Fig. 2B). Although CT-B-labeled neurons were observed throughout the rostrocaudal extension of the 
PAG columns (i.e., rostral, intermediate, and caudal PAG), the majority of the projection neurons for all experimental cases were located in the intermediate and caudal aspects of the respective PAG columns (data not shown).

\subsection{Distribution of retrogradely labeled neurons in ponto-medullary respiratory} nuclei after CT-B injections in the lateral and ventrolateral columns of the PAG

The locations of CT-B injections in the IPAG and vIPAG are illustrated in Fig 3A. Overall, our analysis revealed the highest number of CT-B-labeled neurons in the KFn, in comparison to the medullary BötC, pre-BötC, or caudal raphé ( $p<0.05$; Fig. 3B, C). Specifically, injections in the PAG revealed on average $48 \pm 6 \mathrm{n} / \mathrm{c}$ in the $\mathrm{KFn}$, versus only $6 \pm 1 \mathrm{n} / \mathrm{c}$ in the pre-BötC, $5 \pm 2 \mathrm{n} / \mathrm{c}$ in the BötC, or $1 \pm 1 \mathrm{n} / \mathrm{c}$ in the caudal raphé. Analysis of the laterality of retrogradely neurons in the pontine KFn indicated the highest number of CT-B-labeled neurons located

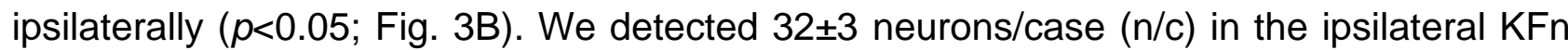
and $15 \pm 4 \mathrm{n} / \mathrm{c}$ in the contralaterally. In the medullary pre-BötC, we observed a tendency of higher number of CT-B-labeled neurons in the ipsilateral hemisphere. We detected $5 \pm 1 \mathrm{n} / \mathrm{c}$ located ipsilaterally and $1 \pm 1 \mathrm{n} / \mathrm{c}$ contralaterally (Fig. 3B). Finally, in the BötC, we detected $2 \pm 1 \mathrm{n} / \mathrm{c}$ in the ipsilateral hemisphere, and $3 \pm 1 \mathrm{n} / \mathrm{c}$ in the contralaterally (not significant; Fig. 3B). Please note that relative proportion of efferent versus afferent projections is an order of magnitude different because the entirety of a PAG column was not covered by a single CTB injection, whereas all cells within a PAG column were counted when quantifying efferent projections. Given that the injection volumes were of a consistent volume, and were targeted toward the caudal aspect of the PAG in the case of afferent projections (see Fig 3A), their relative proportions should still be a reliable measure for comparison.

\subsection{Summary of the afferent and efferent connectivity of lateral and ventrolateral PAG} columns with respiratory nuclei in the ponto-medullary brainstem

The topographical organization of the descending and ascending PAG projections is summarized in a network connectivity graph (Figure 4). The graph shows the relative 
proportion of efferent projections from the midbrain PAG columns to each ponto-medullary target nuclei (Fig. 4A), and the afferent inputs to the PAG arising from the downstream targets (Fig. 4B). The functional implications of the reciprocal connectivity of the PAG in the context of volitional control of breathing are discussed below. 


\section{Discussion}

The midbrain PAG is thought to play a critical role in modulating breathing via inputs to the ponto-medullary respiratory network. However, PAG anatomical connectivity with core nuclei of the respiratory network remains vague. The present study identified neurons in the PAG that target either the pontine KFn, the caudal raphé, or the medullary pre-BötC, but not the BötC. It is likely that these descending PAG neurons are important for the volitional modulation or appropriation of respiratory motor activities. Several studies reported that stimulations of the PAG (IPAG and vIPAG, especially) evoke strong respiratory modulations and, in particular, trigger vocalizations (Magoun et al., 1937; Jürgens and Pratt, 1979, Larson and Kistler, 1984; Jürgens, 1994; Zhang et al., 1994; Davis and Zhang, 1996; Huang et a., 2000; Hayward et al., 2003; Zhang et al., 2007; Subramanian et al., 2008b; Jürgens, 2009; Zhang et al., 2009; Subramanian et al., 2013; Subramanian and Holstege, 2013; Farmer et al., 2014). Furthermore, changes in respiratory activity (i.e., an increase in the respiratory frequency) can also be correlated with defensive behaviors (e.g., fight and flight, freezing, aggressive or escape actions) that are elicited by PAG stimulation (Bandler, 1982; Bandler et al., 1985; Bandler and Depaulis, 1991; Carrive et al., 1997; Fanselow, 1991; Walker and Carrive, 2003; Hayward et al., 2003). The present study identified approximately equal numbers of PAG neurons projecting to the KFn, pre-BötC, and caudal raphé nuclei, which have different functions in respiratory pattern formation ( $K F n$; see Dutschmann and Herbert, 2006; Smith et al., 2007; Mörschel and Dutschmann, 2009), rhythm generation (pre-BötC; see Smith et al., 1991; Wenninger et al., 2004; Feldman and Del Negro, 2006), and modulation of the excitability of respiratory network neurons (caudal raphé; see Lindsey et al., 1998; Richter et al., 2003, Hodges and Richerson; 2010). Taken together, our anatomical finding suggests that the PAG may engage a widely distributed network of brainstem nuclei to modulate respiratory motor pattern. 
It has been proposed that the PAG might be the final common pathway mediating changes in the eupneic respiratory motor pattern in the context of various volitional and emotional behaviors (Subramanian and Holstege, 2014), including vocalization (Jürgens and Richter, 1986; Holstege, 1989; Jürgens 1994; Jürgens 2002; Düsterhöft et al., 2004; Subramanian and Holstege, 2009). However, the proposed descending pathway that connects the PAG output with laryngeal and spinal respiratory motor neurons was via solely on PAG projections to the nucleus retroambiguus (NRA) in the caudal medulla (Holstege et al., 1989; Holstege et al., 1997; Vanderhorst et al., 2000). In the PAG-NRA hypothesis, the NRA subsequently acts as an interface that distributes PAG signals to laryngeal motor neurons of the nucleus ambiguus (Vanderhorst et al., 2001) and various respiratory motor pools in the spinal cord (Vanderhorst and Holstege, 1995; Holstege et al., 1997), bypassing the respiratory rhythm and pattern generating network in the ponto-medullary brainstem, and is thought to be critical for PAG-mediated modulation of breathing, particularly during vocalization (Zhang et al., 1995; Subramanian and Holstege, 2009). However, recent work from our laboratory has shown that the eupneic respiratory motor pattern is generated by an anatomically widely-distributed brainstem network (Dhingra et al., 2019a,b; Dhingra et al., 2020). Consistent with these observations, the present study shows that the IPAG and vIPAG send multiple descending projections to these widely-distributed and functionally diverse brainstem respiratory control nuclei. For instance, in line with a previous study, the PAG receives afferents from the respiratory rhythm generating circuits of the pre-BötC (Yang and Feldman, 2018), and, therefore, may receive information regarding the respiratory rhythm. However, compared to pre-BötC, we observed that the KFn has significantly stronger afferent inputs to the PAG. Thus, the IPAG and VIPAG columns reciprocally connected with the KFn, which also receives substantial afferent input from the NRA (Jones et al., 2016). 
In a recent study of the descending forebrain projections targeting the brainstem nuclei important for breathing control, we reported efferent cortical projections predominantly target the PAG and KFn (Trevizan-Baú et al., 2020). The reciprocal connectivity of the PAG and NRA with the KFn seems a coherent anatomical structure for the mediation of volitional respiratory modulation (e.g., vocalization and sniffing; see Trevizan-Baú et al., 2020), which requires control of expiratory airflow via glottal adduction during the post-inspiratory phase (Dutschmann et al., 2014). In contrast, changes in inspiratory frequency associated with emotion (Homma and Masuko, 2008) or behavior (Subramanian et al., 2008a, b; Subramanian and Holstege, 2014; Faul et al., 2019) might be mediated via forebrain to PAG projections (this study; Trevizan-Baú et al., 2020) targeting the pre-inspiratory rhythm generating circuit of the pre-BötC (Subramanian et al., 2013).

In conclusion, the midbrain PAG projects to the ponto-medullary respiratory network (i.e., pontine KFn, medullary pre-BötC, and the caudal raphé), and receives ascending projections from those respiratory control areas, especially from the KFn. The reciprocal PAG-KFn connectivity provides an anatomical framework for the regulation of upper-airway patency during the mediation of breathing modulation in the context of volitional and emotional behaviors. 


\section{Experimental procedures}

\subsection{Animals}

Adult Sprague-Dawley rats of either sex ( $n=18$, weight range: $280-350 \mathrm{~g})$ were used. Rats were housed under a $12: 12 \mathrm{~h}$ light/dark cycle, with free access to lab chow (Ridley Corporation Limited, Australia) and water. All experimental procedures were approved by the Florey Institute of Neuroscience and Mental Health Animal Ethics Committee and performed in accordance with the National Health and Medical Research Council of Australia code of practice for the use of animals for scientific purposes.

\subsection{Surgery}

Details of the full surgical procedures are published in a recent study (Trevizan-Baú et al., 2020) In brief, after craniotomy, a $1 \mu$ l Hamilton syringe (25s-gauge needle) filled with 150nL of $1 \%$ CT-B (1 mg/mL; Invitrogen, OR, USA) was used to pressure inject the retrograde tracer (150nL; $20 \mathrm{~nL} / \mathrm{min}$ ) unilaterally (left side of the brain) in the $\mathrm{KFn}$, BötC, pre-BötC, caudal raphé nuclei, or PAG. We used the following coordinates (Paxinos and Watson, 2007): KFn: -9 mm relative to bregma (A/P), $2.6 \mathrm{~mm}$ lateral to the midline (M/L), and $6.5 \mathrm{~mm}$ ventral to the dorsal surface of the brain (D/V); BötC: $-9 \mathrm{~mm} \mathrm{~A} / \mathrm{P}, 2 \mathrm{~mm} \mathrm{M} / \mathrm{L}$, and $9.5 \mathrm{~mm}$ D/V; pre-BötC: $-9.5 \mathrm{~mm} \mathrm{A/P,} 2 \mathrm{~mm} \mathrm{M/L}$, and $9.5 \mathrm{~mm} \mathrm{D/V;} \mathrm{caudal} \mathrm{raphé} \mathrm{nuclei:}-8.7 \mathrm{~mm} \mathrm{~A} / \mathrm{P}$, $0 \mathrm{~mm} \mathrm{M} / \mathrm{L}$, and $9.4 \mathrm{~mm} \mathrm{D/V}$; and PAG: $-8 \mathrm{~mm} \mathrm{~A} / \mathrm{P}, 0.8 \mathrm{~mm} \mathrm{M} / \mathrm{L}$, and $4.8 \mathrm{~mm} \mathrm{D/V}$. Injections in the medullary regions (i.e., BötC, pre-BötC, and caudal raphé), required an adaptation and the syringe was angled at $22^{\circ}$ backwards from vertical to avoid bleeding from superficial cerebral blood sinuses. After injections were completed, the syringe remained in the brain tissue for $10 \mathrm{~min}$ and was withdraw at $1 \mathrm{~mm} / \mathrm{min}$ to minimize non-specific spread of the tracer along the injection tract (Finkelstein et al, 2000).

\subsection{Tissue preparation and immunohistochemistry}

The full experimental protocols for the immunohistochemistry are published in Trevizan-Baú et al. (2020). In brief ,12-14 days post-injections, rats were transcardially perfused with 
paraformaldehyde (PFA, Sigma-Aldrich) containing $0.2 \%$ picric acid (Sigma) diluted in 0.16 M phosphate buffer (Merck KGaA, Darmstadt, Germany; $\mathrm{pH} 7.2$, initially at $37{ }^{\circ} \mathrm{C}$ and subsequently at $4^{\circ} \mathrm{C}$ ). After postfixation with PFA/picric acid solution for 90 min at $4^{\circ} \mathrm{C}$, the brains were immersed for $72 \mathrm{~h}$ in $0.1 \mathrm{M}$ phosphate buffer $(\mathrm{pH} 7.4)$ containing $15 \%$ sucrose, 0.01\% sodium aside (Sigma) and $0.02 \%$ bacitracin (Sigma) for cryoprotection. Before brain were sectioned with a cryostat (Leica CM1850, Leica Microsystems; coronal sections of $40 \mu \mathrm{m}$ thickness), a small superficial incision was made from the midbrain to the brainstem to distinguish the ipsilateral and contralateral sides of the brain relative to the injection site. Free-floating sections were washed in $0.01 \mathrm{M}$ PBS, followed by incubation in hydrogen peroxide for $20 \mathrm{~min}$ and incubated for $24 \mathrm{~h}$ at room temperature (RT) with a goat anti-CT-B antibody (1:10,000 List Biological Laboratories, CA, USA). Then, sections were washed in $0.01 \mathrm{M}$ PBS, blocked with $5 \%$ normal donkey serum (NDS) in $0.01 \mathrm{M}$ PBS for $1 \mathrm{~h}$ at RT, incubated in the corresponding secondary antibody (anti-sheep biotin, Jackson ImmunoResearch Laboratories, West Grove, PA; 1:500 in 0.01 M PBS) for 1h at RT, and subsequently incubated in an ABC kit (Vectastain ${ }^{8}$ Elite ABC-HRP Kit) for $1 \mathrm{~h}$ at RT. Finally, sections were incubated in diaminobenzidine (DAB) substrate (1:10, Roche Diagnostics Mannheim, Germany) for $4 \mathrm{~min}$, followed by $1 \% \mathrm{H}_{2} \mathrm{O}_{2}$ incubation for 4 min. After several washing steps, the sections were then mounted on slides and cover-slipped.

\subsection{Data analysis and image processing}

To identify and document the location of the midbrain and brainstem injection sites, we used a brightfield microscope (Leica Biosystems). Injection sites were photographed and represented on a schematic drawing (Fig. 1A; Fig. 3A). Additional information regarding the distribution and location of the injection sites are detailed in our previous study (TrevizanBaú et al., 2020).

For analysis, we quantified the number of CT-B-labeled neurons in the brain region of interest. CT-B-labeled neurons were recognized by the presence of black punctate 
granules in the neuronal cell bodies (somata) and dendrites, and the absence of stained cell nuclei (for details, see Trevizan-Baú et al., 2020). Cell counts were analyzed with GraphPad Prism (version 7.02; GraphPad Software; San Diego, CA). Data were analyzed by One-way ANOVA followed by Tukey's multiple comparison test to compare the number of CT-Blabeled neurons between the target regions. To determine statistical significance between the numbers of CT-B-labeled neurons in the ipsilateral and contralateral hemisphere, data were analyzed by paired t-test (parametric). All values are presented as mean \pm standard error of the mean (SEM), and p-values less than 0.05 were considered statistically significant.

A digital camera (Leica DFC7000T) mounted to the microscope (Leica DM6B LED) was used to take representative images. To best represent sections viewed under the microscope, we used the Adobe Photoshop CC19 software (Adobe Systems Inc., San Jose, CA) to optimize brightness and contrast of the digital images, as well as to assemble representative images. 


\section{Acknowledgements}

We acknowledge that this work was conducted on the traditional land of the Wurundjeri people of the Kulin nation. We pay our respect to their elders past, present and emerging. This work was supported by research grants from the National Health and Medical Research Council of Australia (APP1165529 to MD \& DS, and APP1078943 to SM); and Australian Research Council (Discovery project DP170104861); PT-B is funded by Melbourne Research Scholarship (University of Melbourne; 181858).

\section{Author contributions}

PT-B, DS and MD conceived and designed the experiments. P-TB and MD analyzed the data and prepared figures and tables. PT-B conducted all experiments. All authors contributed to interpretation of the data, reviewed drafts of the manuscript, and approved the final draft.

\section{Conflict of interest}

SBM reports personal payments from Merck and NeRRe Therapeutics and grant income from Merck, for activities outside the scope of this study. All other authors report no conflicts of interest. 


\section{References}

Bandler, R.,1982. Induction of 'page' following microinjections of glutamate into midbrain but not hypothalamus of cats. Neurosci. Lett. 30(2), 183-188. https://doi.org/10.1016/0304-3940(82)90294-4.

Bandler, R., Prineas, S., McCulloch, T., 1985. Further localization of midbrain neurones mediating the defence reaction in the cat by microinjections of excitatory amino acids. Neurosci. Lett. 56(3), 311-316. https://doi.org/10.1016/0304-3940(85)90261-7.

Bandler, R., Carrive, P., Depaulis, A., 1991. Emerging principles of organization of the midbrain periaqueductal gray matter. In The midbrain periaqueductal gray matter (pp. 1-8). Springer, Boston, MA. https://doi.org/10.1007/978-1-4615-3302-3_1.

Bandler, R., Depaulis, A., 1991. Midbrain periaqueductal gray control of defensive behaviour in the cat and the rat. In The midbrain periaqueductal gray matter (pp. 175198). Springer, Boston, MA. https://doi.org/10.1007/978-1-4615-3302-3_11.

Beitz, A. J., Mullett, M. A., Weiner, L. L., 1983. The periaqueductal gray projections to the rat spinal trigeminal, raphe magnus, gigantocellular pars alpha and paragigantocellular nuclei arise from separate neurons. Brain Res. 288(1-2), 307314. https://doi.org/10.1016/0006-8993(83)90108-7.

Buzsáki, G., 2010. Neural syntax: cell assemblies, synapsembles, and readers. Neuron. 68(3), 362-385. https://doi.org/10.1016/j.neuron.2010.09.023.

Carrive, P., Bandler, R., Dampney, R. A. L., 1988. Anatomical evidence that hypertension associated with the defence reaction in the cat is mediated by a direct projection from a restricted portion of the midbrain periaqueductal grey to the subretrofacial nucleus of the medulla. Brain Res. 460(2), 339-345. https://doi.org/10.1016/00068993(88)90378-2.

Carrive, P., Bandler, R., Dampney, R. A. L., 1989. Viscerotopic control of regional vascular beds by discrete groups of neurons within the midbrain periaqueductal gray. Brain Res. 493(2), 385-390. https://doi.org/10.1016/0006-8993(89)91176-1.

Carrive, P., 1993. Behav. Brain Res. 58(1-2), 27-47. https://doi.org/10.1016/01664328(93)90088-8.

Carrive, P., Leung, P., Harris, J., Paxinos, G., 1997. Conditioned fear to context is associated with increased Fos expression in the caudal ventrolateral region of the midbrain periaqueductal gray. Neuroscience. 78(1), 165-177. https://doi.org/10.1016/S0306-4522(97)83047-3.

Cowie, R. J., Holstege, G., 1992. Dorsal mesencephalic projections to pons, medulla, and spinal cord in the cat: limbic and non-limbic components. J. Comp. Neurol. 319(4), 536-559. https://doi.org/10.1002/cne.903190406.

Dampney, R. A., Furlong, T. M., Horiuchi, J., ligaya, K., 2013. Role of dorsolateral periaqueductal grey in the coordinated regulation of cardiovascular and respiratory function. Auton. Neurosci. 175(1-2), 17-25. https://doi.org/10.1016/j.autneu.2012.12.008.

Dhingra, R. R., Furuya, W. I., Bautista, T. G., Dick, T. E., Galán, R. F., Dutschmann, M., 2019a. Increasing local excitability of brainstem respiratory nuclei reveals a distributed network underlying respiratory motor pattern formation. Front. Physiol. 10, 887. https://doi.org/10.3389/fphys.2019.00887.

Dhingra, R. R., Furuya, W. I., Galán, R. F., Dutschmann, M., 2019b. Excitation-inhibition balance regulates the patterning of spinal and cranial inspiratory motor outputs in rats in situ. Resp. Physiol. Neurobi. 266, 95-102. https://doi.org/10.1016/j.resp.2019.05.001. 
Dhingra, R. R., Dick, T. E., Furuya, W. I., Galán, R. F., Dutschmann, M., 2020. Volumetric mapping of the functional neuroanatomy of the respiratory network in the perfused brainstem preparation of rats. J. Physiol. https://doi.org/10.1113/JP279732.

Düsterhöft, F., Häusler, U., Jürgens, U., 2004. Neuronal activity in the periaqueductal gray and bordering structures during vocal communication in the squirrel monkey. Neuroscience. 123(1), 53-60. https://doi.org/10.1016/j.neuroscience.2003.07.007.

Dutschmann, M., Herbert, H., 2006. The Kölliker-Fuse nucleus gates the postinspiratory phase of the respiratory cycle to control inspiratory off-switch and upper airway resistance in rat. Eur. J. Neurosci. 24(4), 1071-1084. https://doi.org/10.1111/j.14609568.2006.04981.x.

Dutschmann, M., Dick, T. E, 2012. Pontine mechanisms of respiratory control. Compr. Physiol. 2(4), 2443-2469. https://doi.org/10.1002/cphy.c100015.

Dutschmann, M., Jones, S. E., Subramanian, H. H., Stanic, D., Bautista, T. G., 2014. The physiological significance of postinspiration in respiratory control. In Prog. Brain Res. 212, pp. 113-130. Elsevier. https://doi.org/10.1016/B978-0-444-63488-7.00007-0.

Davis, P. J., Zhang, S. P., 1996. Midbrain and medullary regulation of respiration and vocalization. In Prog. Brain Res. 107, pp. 315-325. Elsevier. https://doi.org/10.1016/S0079-6123(08)61873-7.

Fanselow, M. S., 1991. The midbrain periaqueductal gray as a coordinator of action in response to fear and anxiety. In The midbrain periaqueductal gray matter. pp. 151173). Springer, Boston, MA. https://doi.org/10.1007/978-1-4615-3302-3_10.

Farmer, D. G., Bautista, T. G., Jones, S. E., Stanic, D., Dutschmann, M., 2014. The midbrain periaqueductal grey has no role in the generation of the respiratory motor pattern, but provides command function for the modulation of respiratory activity. Resp. Physiol. Neurobi. 204, 14-20. https://doi.org/10.1016/j.resp.2014.07.011.

Faull, O. K., Subramanian, H. H., Ezra, M., Pattinson, K. T., 2019. The midbrain periaqueductal gray as an integrative and interoceptive neural structure for breathing. Neurosci. Biobeh. R. 98, 135-144.

https://doi.org/10.1016/j.neubiorev.2018.12.020.

Feldman, J. L., Del Negro, C. A., 2006. Looking for inspiration: new perspectives on respiratory rhythm. Nat. Rev. Neurosci. 7, 232-241. https://doi.org/10.1038/nrn1871.

Finkelstein, D. I., Stanic, D., Parish, C. L., Tomas, D., Dickson, K., Horne, M. K., 2000. Axonal sprouting following lesions of the rat substantia nigra. Neuroscience, 97, 99112. https://doi.org/10.1016/s0306-4522(00)00009-9.

Hayward, L. F., Swartz, C. L., Davenport, P. W., 2003. Respiratory response to activation or disinhibition of the dorsal periaqueductal gray in rats. J. Appl. Physiol. 94(3), 913922. https://doi.org/10.1152/japplphysiol.00740.2002.

Huang, Z. G., Subramanian, S. H., Balnave, R. J., Turman, A. B., Chow, C. M., 2000. Roles of periaqueductal gray and nucleus tractus solitarius in cardiorespiratory function in the rat brainstem. Resp. Physiol. 120(3), 185-195. https://doi.org/10.1016/S0034-5687(00)00107-9.

Hodges, M. R., Richerson, G. B., 2010. The role of medullary serotonin (5-HT) neurons in respiratory control: contributions to eupneic ventilation, $\mathrm{CO} 2$ chemoreception, and thermoregulation. J. Appl. Physiol. 108(5), 1425-1432. https://doi.org/10.1152/japplphysiol.01270.2009.

Holstege, G., 1989. Anatomical study of the final common pathway for vocalization in the cat. J. Comp. Neurol. 284(2), 242-252. https://doi.org/10.1002/cne.902840208.

Holstege, G., 1991. Descending pathways from the periaqueductal gray and adjacent areas. In The midbrain periaqueductal gray matter: functional anatomical and 
immunohistochemical organization (Depaulis A, Bandler R, eds), 239-265. Springer, Boston, MA. https://doi.org/10.1007/978-1-4615-3302-3_14.

Holstege, G., Kerstens, L., Moes, M. C., VanderHorst, V. G. J. M., 1997. Evidence for a periaqueductal gray-nucleus retroambiguus-spinal cord pathway in the rat. Neuroscience. 80(2), 587-598. https://doi.org/10.1016/S0306-4522(97)00061-4.

Homma, I., Masaoka, Y., 2008. Breathing rhythms and emotions. Exp. Physiol. 93(9), 1011-1021. https://doi.org/10.1113/expphysiol.2008.042424.

Jürgens, U., Pratt, R., 1979. Role of the periaqueductal grey in vocal expression of emotion. Brain research. https://doi.org/10.1016/0006-8993(79)90830-8.

Jürgens, U., Richter, K., 1986. Glutamate-induced vocalization in the squirrel monkey. Brain Res. 373(1-2), 349-358. https://doi.org/10.1016/0006-8993(86)903495.

Jürgens, U., 1994. The role of the periaqueductal grey in vocal behaviour. Behav. Brain Res. 62(2), 107-117. https://doi.org/10.1016/0166-4328(94)90017-5.

Jürgens, U., 2002. A study of the central control of vocalization using the squirrel monkey. Med. Eng. Phys. 24(7-8), 473-477. https://doi.org/10.1016/S13504533(02)00051-6.

Jürgens, U., 2009. The neural control of vocalization in mammals: a review. J. Voice. 23(1), 1-10. https://doi.org/10.1016/j.jvoice.2007.07.005.

Jones, S. E., Saad, M., Lewis, D. I., Subramanian, H. H., Dutschmann, M., 2012. The nucleus retroambiguus as possible site for inspiratory rhythm generation caudal to obex. Resp. Physiol. Neurobi. 180(2-3), 305-310. https://doi.org/10.1016/j.resp.2011.12.007.

Jones, S. E., Stanić, D., Dutschmann, M., 2016a. Dorsal and ventral aspects of the most caudal medullary reticular formation have differential roles in modulation and formation of the respiratory motor pattern in rat. Brain Struct. Funct. 221(9), 43534368. https://doi.org/10.1007/s00429-015-1165-x.

Jones, S. E., Dutschmann, M., 2016b. Testing the hypothesis of neurodegeneracy in respiratory network function with a priori transected arterially perfused brain stem preparation of rat. J. Neurophysiol. 115(5), 2593-2607. https://doi.org/10.1152/jn.01073.2015.

Krout, K. E., Jansen, A. S., Loewy, A. D., 1998. Periaqueductal gray matter projection to the parabrachial nucleus in rat. J. Comp. Neurol. 401(4), 437-454. https://doi.org/10.1002/(SICI)1096-9861(19981130)401:4\%3C437::AIDCNE2\%3E3.0.CO;2-5.

Larson, C. R., Kistler, M. K., 1984. Periaqueductal gray neuronal activity associated with laryngeal EMG and vocalization in the awake monkey. Neurosci. Lett. 46(3), 261266. https://doi.org/10.1016/0304-3940(84)90109-5.

Lindsey, B. G., Arata, A., Morris, K. F., Hernandez, Y. M., Shannon, R., 1998. Medullary raphe neurones and baroreceptor modulation of the respiratory motor pattern in the cat. J. Physiol. 512(3), 863-882. https://doi.org/10.1111/j.1469-7793.1998.863bd.x.

Magoun, H. W., Atlas, D., Ingersoll, E. H., Ranson, S. W., 1937. Associated facial, vocal and respiratory components of emotional expression: an experimental study. J. Neurol. Psychopatho. 17(67), 241. 10.1136/jnnp.s1-17.67.241.

Mörschel, M., Dutschmann, M., 2009. Pontine respiratory activity involved in inspiratory/expiratory phase transition. Philos. T. R. Soc. B. 364(1529), 2517-2526. https://doi.org/10.1098/rstb.2009.0074.

Paxinos, G., Watson, C., 2007. The Rat Brain in Stereotaxic Coordinates: Academic Press. 
Richter, D. W., Manzke, T., Wilken, B., Ponimaskin, E., 2003. Serotonin receptors: guardians of stable breathing. Trends. Mol. Med. 9(12), 542-548. https://doi.org/10.1016/j.molmed.2003.10.010.

Smith, J. C., Ellenberger, H. H., Ballanyi, K., Richter, D. W., Feldman, J. L., 1991. PreBotzinger complex: a brainstem region that may generate respiratory rhythm in mammals. Science. 254(5032), 726-729. 10.1126/science.1683005.

Smith, J. C., Abdala, A. P. L., Koizumi, H., Rybak, I. A., Paton, J. F., 2007. Spatial and functional architecture of the mammalian brain stem respiratory network: a hierarchy of three oscillatory mechanisms. J. Neurophysiol. https://doi.org/10.1152/jn.00985.2007.

Subramanian, H. H., Balnave, R. J., Holstege, G., 2008a. The midbrain periaqueductal gray control of respiration. J. Neurosci. 28(47), 12274-12283. https://doi.org/10.1523/JNEUROSCI.4168-08.2008.

Subramanian, H., Huang, Z. G., Balnave, R., 2008b. Responses of brainstem respiratory neurons to activation of midbrain periaqueductal gray in the rat. In Integration in Respiratory Control (pp. 377-381). Springer, New York, NY. https://doi.org/10.1007/978-0-387-73693-8_66.

Subramanian, H. H., Holstege, G., 2009. The nucleus retroambiguus control of respiration. J. Neurosci. 29(12), 3824-3832. https://doi.org/10.1523/JNEUROSCI.0607-09.2009.

Subramanian, H. H., Holstege, G., 2013. Stimulation of the midbrain periaqueductal gray modulates pre-inspiratory neurons in the ventrolateral medulla in the rat in vivo. J. Comp. Neurol. 521(13), 3083-3098. https://doi.org/10.1002/cne.23334.

Subramanian, H. H., Holstege, G., 2014. The midbrain periaqueductal gray changes the eupneic respiratory rhythm into a breathing pattern necessary for survival of the individual and of the species. In Prog. Brain Res. 212, 351-384). https://doi.org/10.1016/B978-0-444-63488-7.00017-3.

Trevizan-Baú, P., Dhingra R.R., Furuya W.I., Stanić, D., Mazzone S.B., Dutschmann, M., 2020. Forebrain projection neurons target functionally diverse respiratory control areas in the midbrain, pons and medulla oblongata. bioRxiv. https://doi.org/10.1101/2020.08.21.260422.

van Bockstaele, E. J., Aston-Jones, G., Pieribone, V. A., Ennis, M., Shipley, M. T., 1991. Subregions of the periaqueductal gray topographically innervate the rostral ventral medulla in the rat. J. Comp. Neurol. 309(3), 305-327. https://doi.org/10.1002/cne.903090303.

Vanderhorst, V. G., Holstege, G., 1995. Caudal medullary pathways to lumbosacral motoneuronal cell groups in the cat: evidence for direct projections possibly representing the final common pathway for lordosis. J. Comp. Neurol. 359(3), 457475. https://doi.org/10.1002/cne.903590308.

Vanderhorst, V. G., Terasawa, E., Ralston III, H. J., Holstege, G., 2000. Monosynaptic projections from the lateral periaqueductal gray to the nucleus retroambiguus in the rhesus monkey: implications for vocalization and reproductive behavior. J. Comp. Neurol. 424(2), 251-268. https://doi.org/10.1002/10969861(20000821)424:2\%3C251::AID-CNE5\%3E3.0.CO;2-D.

Vanderhorst, V. G. J. M., Terasawa, E., Ralston III, H. J., 2001. Monosynaptic projections from the nucleus retroambiguus region to laryngeal motoneurons in the rhesus monkey. Neuroscience. 107(1), 117-125. https://doi.org/10.1016/S03064522(01)00343-8.

Walker, P., Carrive, P., 2003. Role of ventrolateral periaqueductal gray neurons in the behavioral and cardiovascular responses to contextual conditioned fear and poststress recovery. Neuroscience. 116(3), 897-912. https://doi.org/10.1016/S03064522(02)00744-3. 
Wenninger, J. M., Pan, L. G., Klum, L., Leekley, T., Bastastic, J., Hodges, M. R., Feroah, T. R., Davis, S., Forster, H. V., 2004. Large lesions in the pre-Botzinger complex area eliminate eupneic respiratory rhythm in awake goats. J. Appl. Physiol. 97(5), 16291636. https://doi.org/10.1152/japplphysiol.00953.2003.

Yasui, Y., Cechetto, D. F., Saper, C. B., 1990. Evidence for a cholinergic projection from the pedunculopontine tegmental nucleus to the rostral ventrolateral medulla in the rat. Brain Res. 517(1-2), 19-24. https://doi.org/10.1016/0006-8993(90)91002-X.

Yuste, R., 2015. From the neuron doctrine to neural networks. Nat. Rev. Neurosci. 16(8), 487-497. https://doi.org/10.1038/nrn3962.

Zhang, S. P., Davis, P. J., Bandler, R., Carrive, P., 1994. Brain stem integration of vocalization: role of the midbrain periaqueductal gray. J. Neurophysiol. 72(3), 13371356. https://doi.org/10.1152/jn.1994.72.3.1337.

Zhang, S. P., Bandler, R., Davis, P. J., 1995. Brain stem integration of vocalization: role of the nucleus retroambigualis. J. Neurophysiol. 74(6), 2500-2512. https://doi.org/10.1152/jn.1995.74.6.2500.

Zhang, W., Hayward, L. F., Davenport, P. W., 2007. Respiratory responses elicited by rostral versus caudal dorsal periaqueductal gray stimulation in rats. Auton. Neurosci. 134(1-2), 45-54. https://doi.org/10.1016/j.autneu.2007.02.003.

Zhang, W., Hayward, L. F., Davenport, P. W., 2009. Influence of dorsal periaqueductal gray activation on respiratory occlusion reflexes in rats. Auton. Neurosci. 150(1-2), 62-69. https://doi.org/10.1016/j.autneu.2009.04.008. 


\section{CT-B injections in the ponto-medullary respiratory regions}

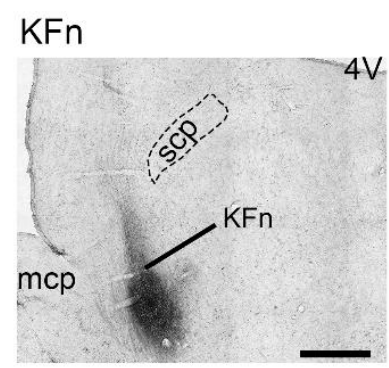

pre-BötC
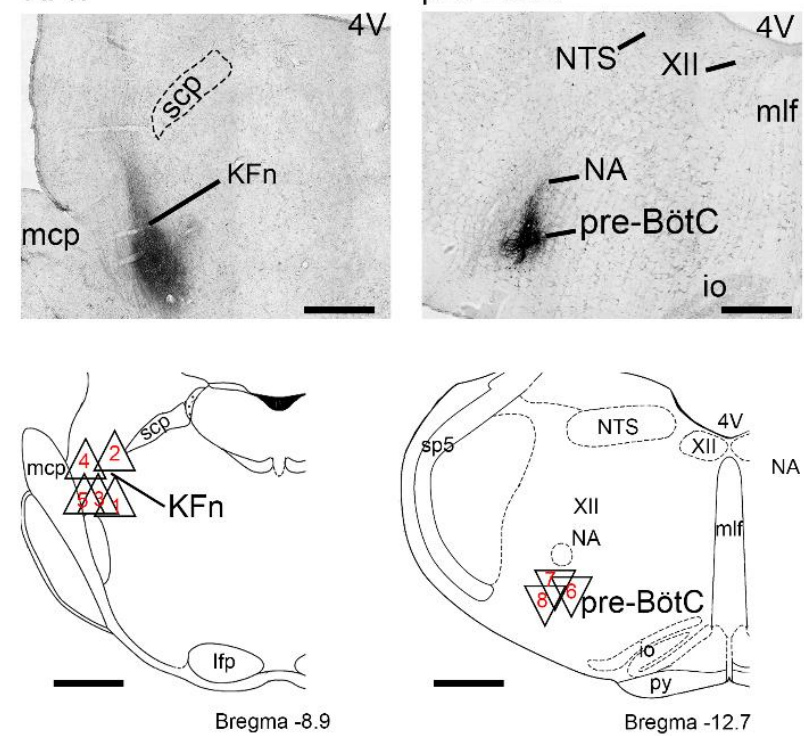

BötC

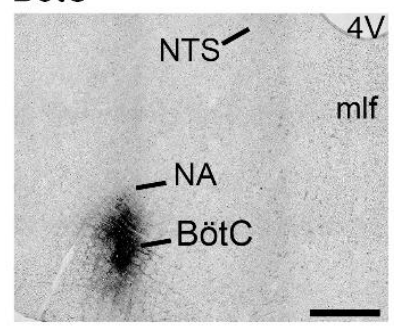

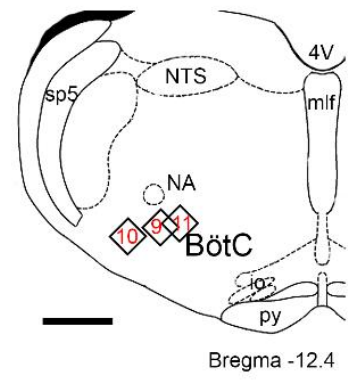

caudal raphé

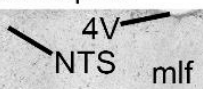

- NA

$\mathrm{RPa}$
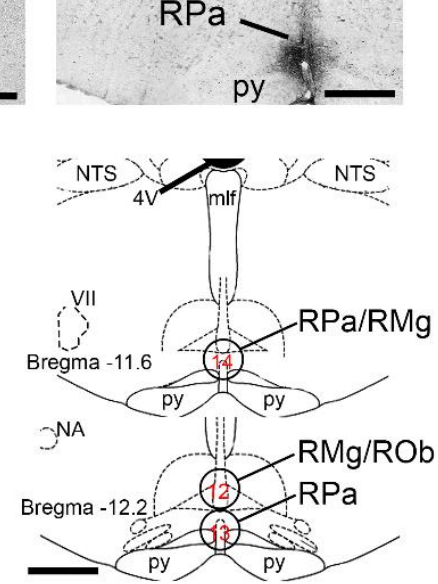

Figure 1. CT-B injections in the pontine Kölliker-Fuse nucleus, the medullary pre-Bötzinger complex and Bötzinger complex, and the caudal raphé. Photomicrographs of representative injections (top) and schematic drawings (bottom) depicting the location of all CT-B injections in the KFn (regular triangle), pre-BötC (upside-down triangle), BötC (square), caudal raphé (circles) relative to bregma. Each number represents a single experimental case. Abbreviations: $4 \mathrm{~V}=$ fourth ventricle; BötC $=$ Bötzinger complex; $\mathrm{KFn}=$ Kölliker-Fuse nucleus; $\mathrm{mcp}=$ middle cerebellar peduncle; io $=$ inferior olive; $\mathrm{mlf}=$ medial longitudinal fasciculus; NA = nucleus ambiguous; NTS = solitary tract nucleus; $P A G=$ periaqueductal gray; pre-BötC =, pre-Bötzinger complex; $p y=$ pyramidal tract; $\mathrm{RMg}=$ raphé magnus; $\mathrm{ROb}$ $=$ raphé obscurus; $\mathrm{RPa}=$ raphé pallidus; $\mathrm{scp}=$ superior cerebellar peduncle; $\mathrm{XII}=$ facial nucleus. Scale bars: $200 \mu \mathrm{m}$. 


\section{A. Number of CT-B-labeled neurons in the PAG}
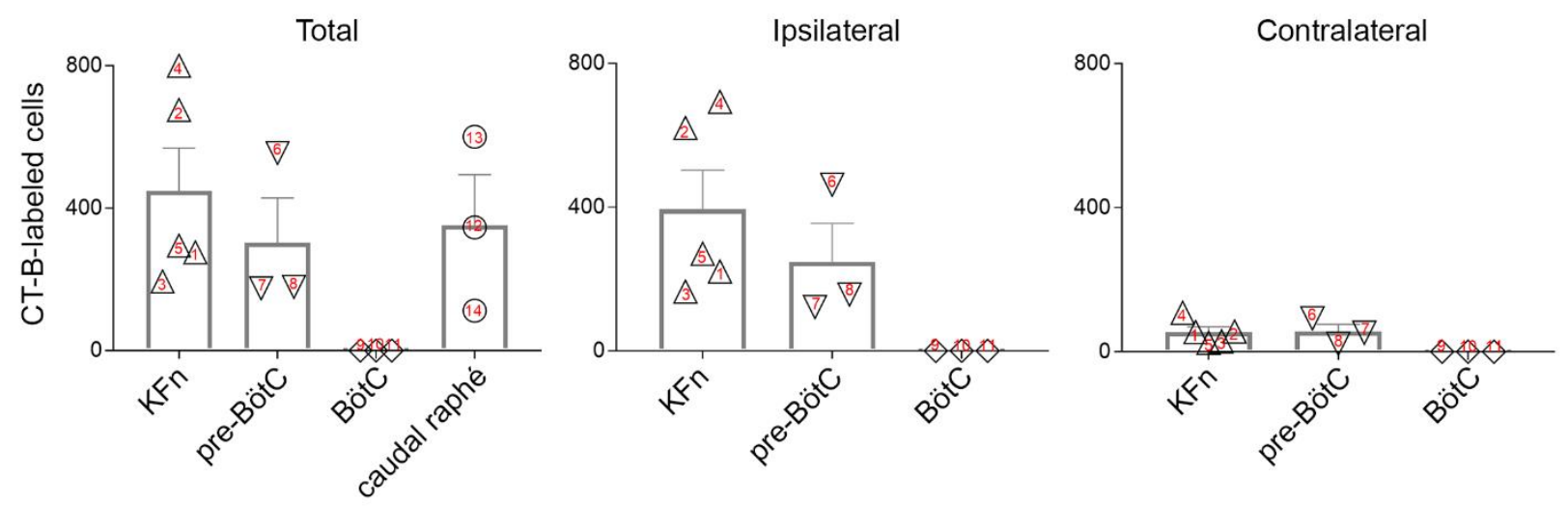

B. Number of CT-B-labeled neurons in the PAG columns
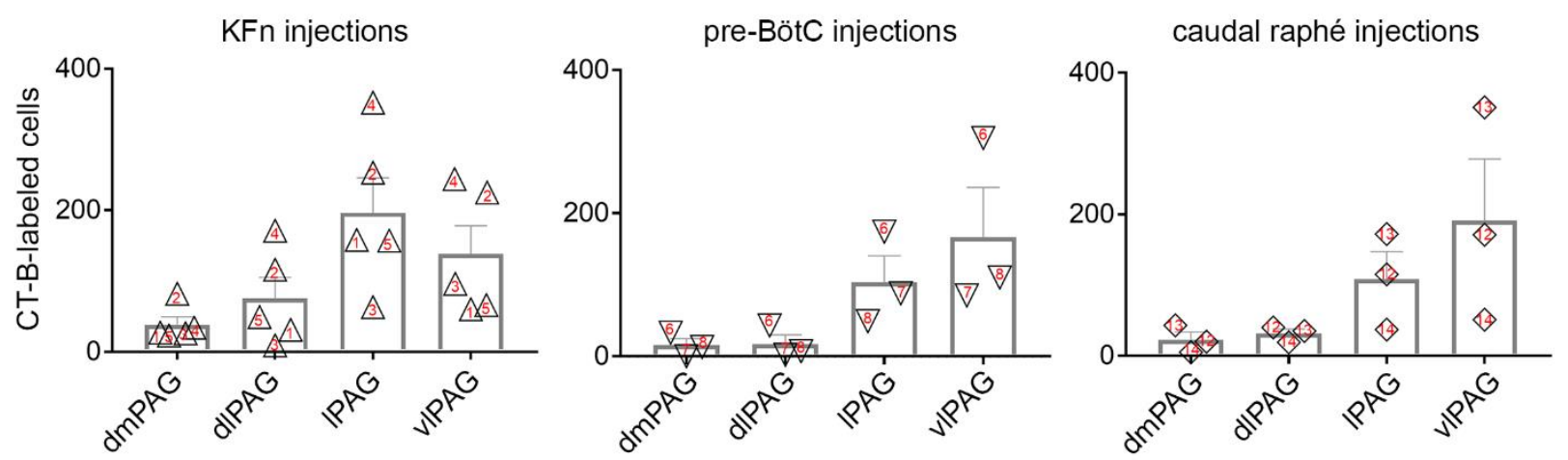

\section{Representative PAG section with CT-B-labeled neurons}
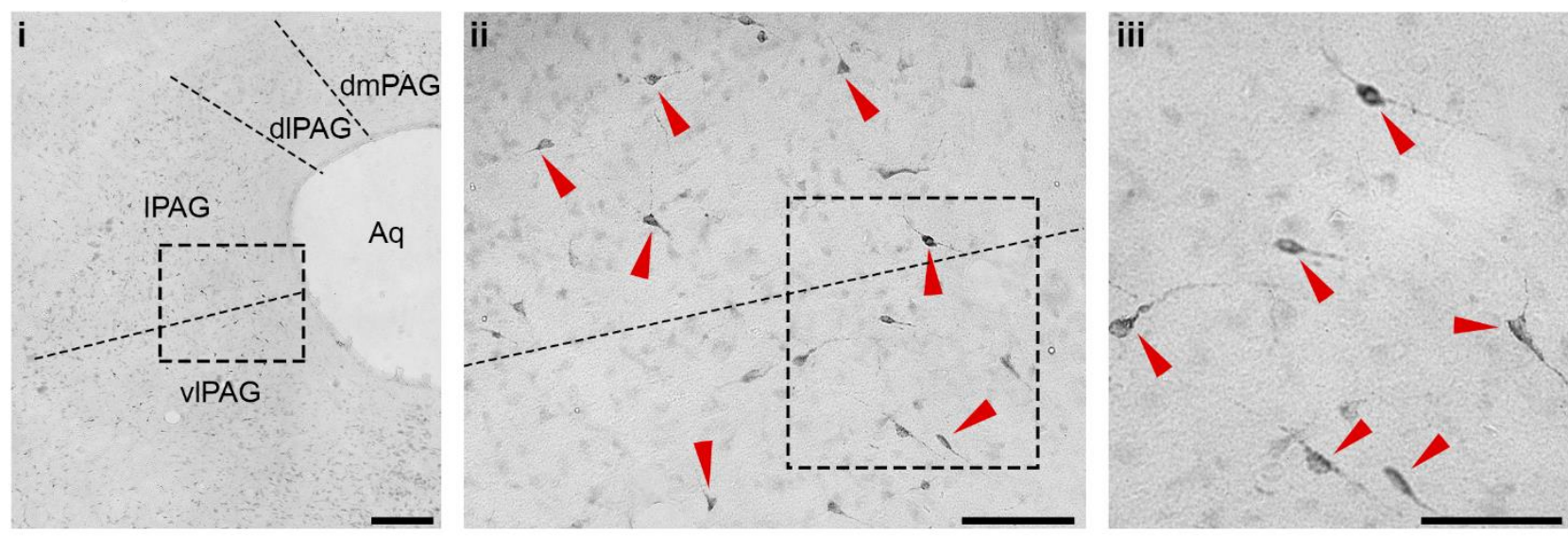

Figure 2. Injections in the ponto-medullary target regions resulted in CT-B-labeled neurons in the midbrain PAG. A: Total, ipsilateral, and contralateral distribution of labeled neurons in the PAG after injections in the KFn, pre-BötC, BötC, or caudal raphé. Analysis of the ipsilateral and contralateral distribution of CT-B-labeled neurons revealed a significant ipsilateral dominance of descending PAG projections targeting the KFn $(p<0.05)$, and a tendency of ipsilateral PAG projections to the pre-BötC. B: Total number of CT-B-labeled neurons in the dmPAG, dIPAG, IPAG and vIPAG after CT-B injections in the pontine KFn, pre-BötC, or caudal raphé. Overall, we observed a predominance of CT-B-labeled neurons in the lateral and ventrolateral columns of the PAG. All values are expressed as mean \pm standard error of the mean. C: Representative images of CT-B-labeled neurons in the PAG: i) the photomicrograph at the lowest magnification shows the retrograde labeled neurons in the context of the PAG columns; ii) at higher magnification (20x), CT-B-labeled neurons (red 
arrowheads) in the IPAG and vIPAG are shown; and iii) at 40x, CT-B-labeled neurons (red arrowheads) is more evident. Abbreviations: $\mathrm{Aq}=$ aqueduct; $\mathrm{BötC}=\mathrm{Bötzinger} \mathrm{complex;} \mathrm{KFn}$ = Kölliker-Fuse nucleus; dIPAG = dorsolateral PAG; dmPAG = dorsomedial PAG; IPAG = lateral PAG; $P A G=$ periaqueductal gray; vIPAG = ventrolateral $P A G ;$ pre-BötC $=$ preBötzinger complex. Scale bars: $100 \mu \mathrm{m}$ (i); $50 \mu \mathrm{m}$ (ii); $25 \mu \mathrm{m}$ (iii). 
A. CT-B injections in the lateral/ventrolateral PAG
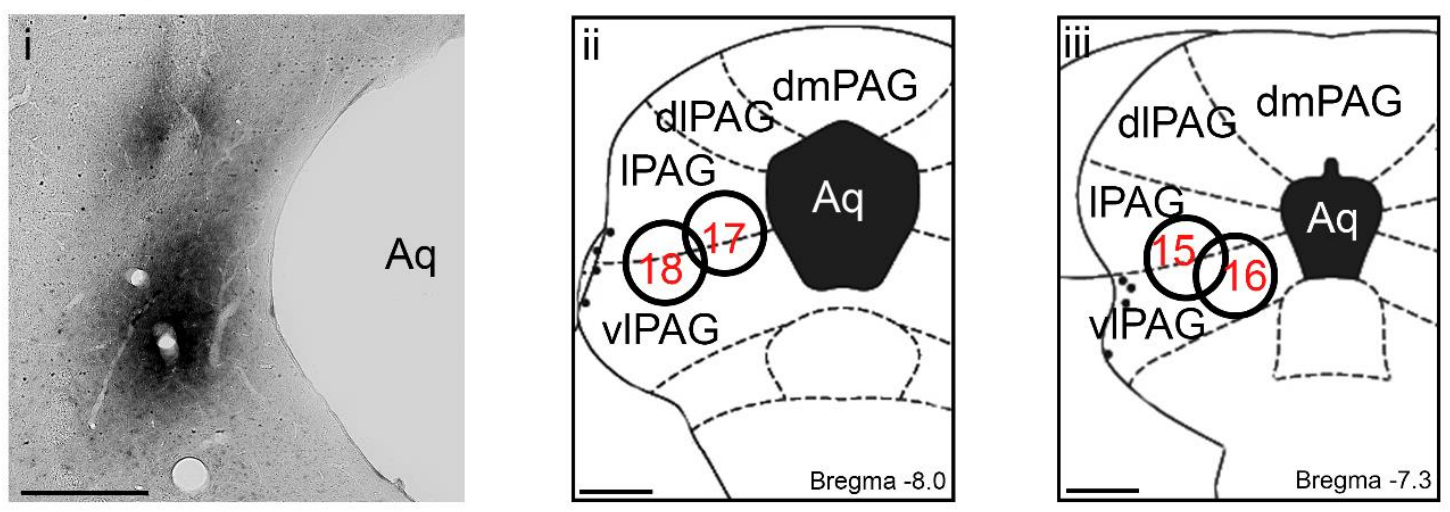

B. Number of CT-B-labeled neurons in the ponto-medullary regions
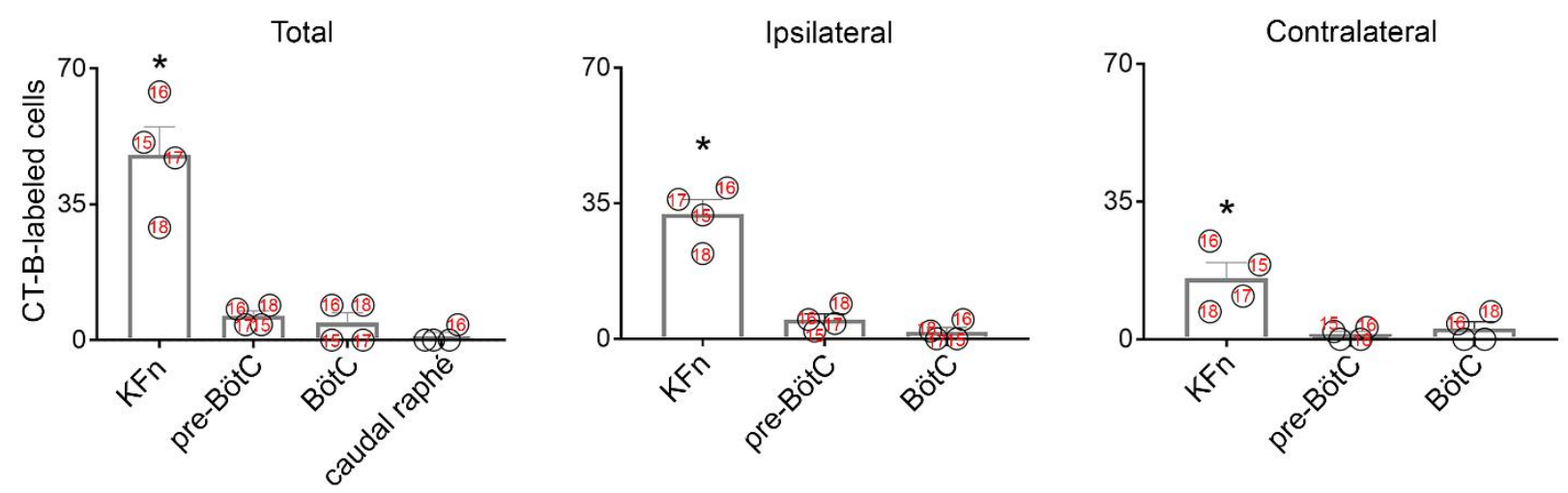

C. Representative images of CT-B-labeled neurons in the ponto-medullary regions
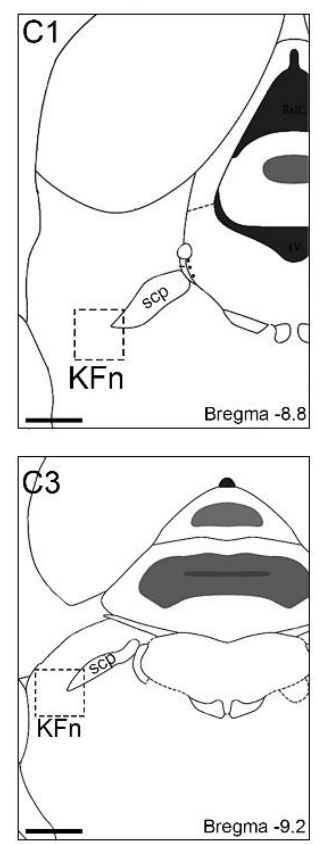
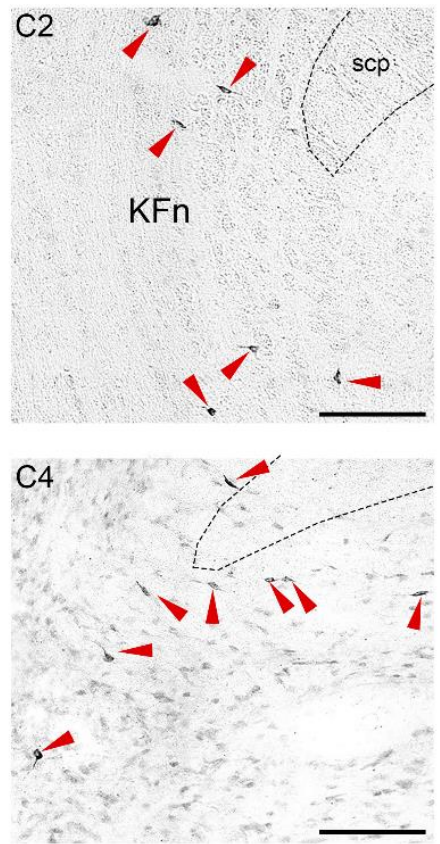
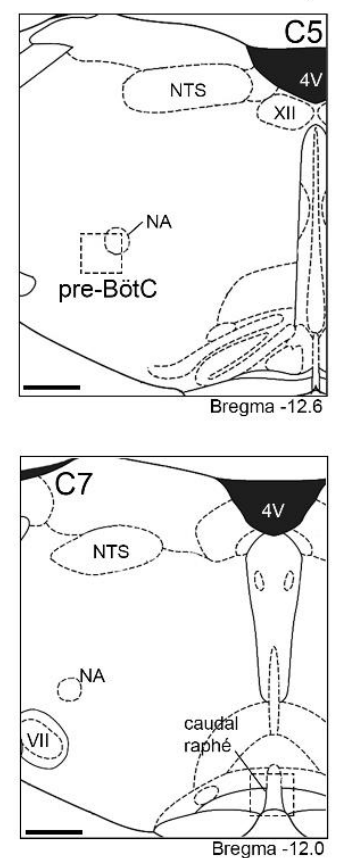

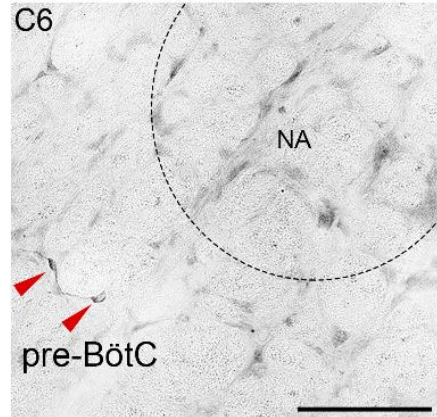

C8

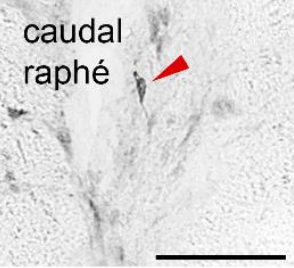

Figure 3. CT-B injections in the lateral/ventrolateral columns of the midbrain PAG resulted in CT-B-labeled neurons in the pontine KFn, the medullary pre-BötC and BötC, and the caudal raphé. A: Photomicrograph of a representative CT-B injection (i) and schematic drawings (ii, iii) depicting the location of all CT-B injections in the PAG (circles). Each number represents a single experimental case. B: Total, ipsilateral, and contralateral cell counts of CT-B-labeled neurons in the KFn, pre-BötC, BötC, and the caudal raphé after injections in the PAG. We observed a greater number of CT-B-labeled neurons in the KFn, compared to pre-BötC, BötC, and caudal raphé. Additionally, our analysis revealed the highest number 
of CT-B-labeled neurons in the ipsilateral KFn $(p<0.05)$. In the medullary pre-BötC, we observed a tendency of higher number of CT-B-labeled neurons ipsilaterally, and no laterality was detected for the BötC. All values are expressed as mean \pm standard error of the mean. C: Representative images of CT-B labeled neurons in the KFn (C1-4), pre-BötC (C5-6), and caudal raphé (C7-8). The photomicrograph on the right $(\mathrm{C} 2, \mathrm{C} 4, \mathrm{C} 6, \mathrm{C} 8 \mathrm{C})$ are of boxed regions marked in $\mathrm{C} 1, \mathrm{C} 3, \mathrm{C} 5$ and $\mathrm{C} 7$, respectively, to show the CT-B-labeled cells. Abbreviations: $4 \mathrm{~V}=$ fourth ventricle; $\mathrm{Aq}=$ aqueduct; $\mathrm{BötC}=$ Bötzinger complex; dIPAG = dorsolateral PAG; dmPAG = dorsomedial PAG; io = inferior olive; KFn = Kölliker-Fuse nucleus; IPAG = lateral PAG; $\mathrm{mcp}=$ middle cerebellar peduncle; mlf = medial longitudinal fasciculus; $N A=$ nucleus ambiguous; NTS = solitary tract nucleus; $P A G$ = periaqueductal gray; pre-BötC = pre-Bötzinger complex; py = pyramidal tract; $s c p=$ superior cerebellar peduncle; vIPAG = ventrolateral PAG; XII = facial nucleus. Scale bars: $400 \mu \mathrm{m}$ (A; C1, C3,

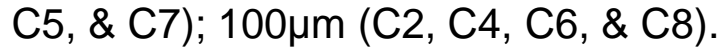


A. PAG inputs to the ponto-medullary respiratory control areas

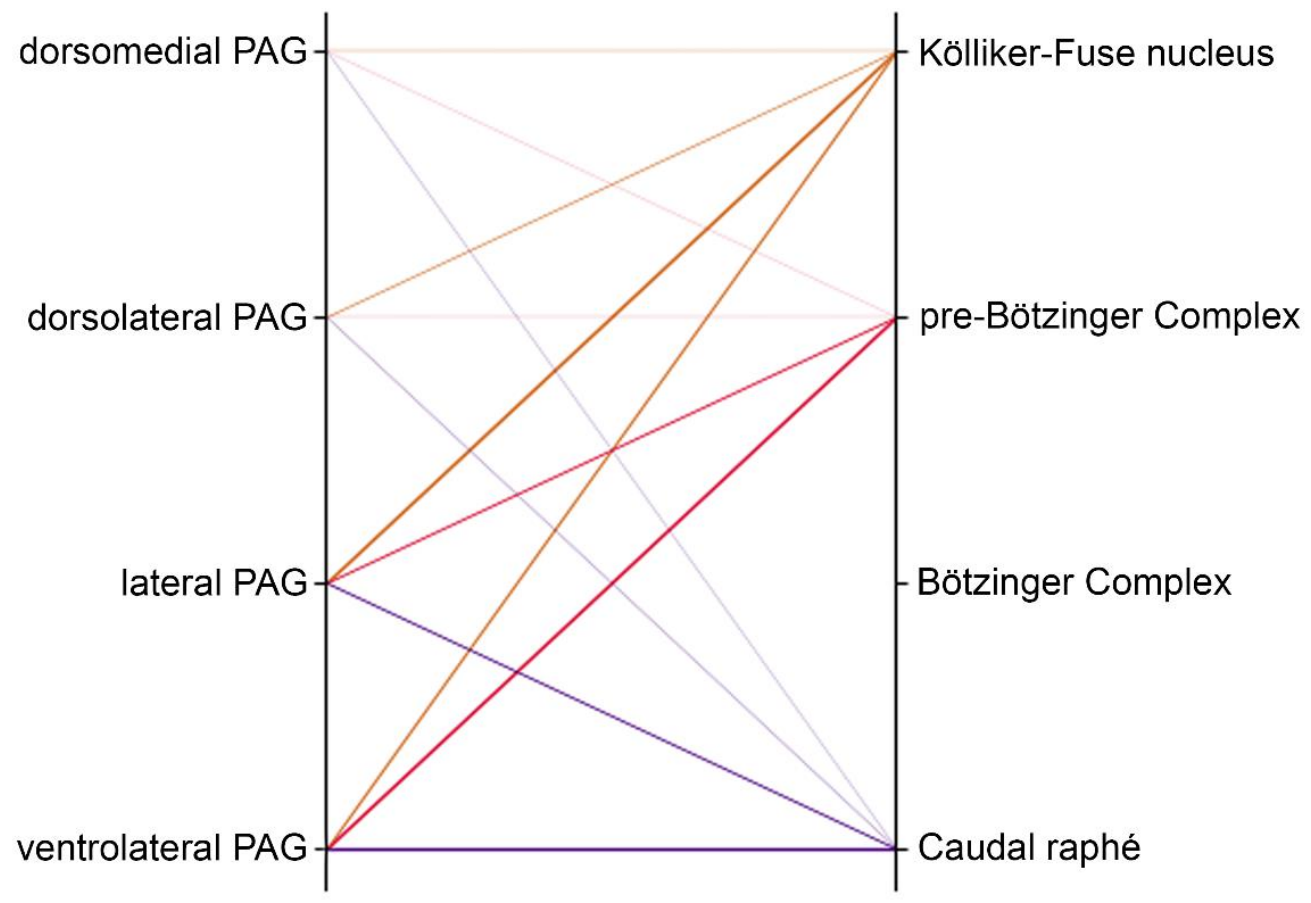

B. Ponto-medullary inputs to the midbrain PAG

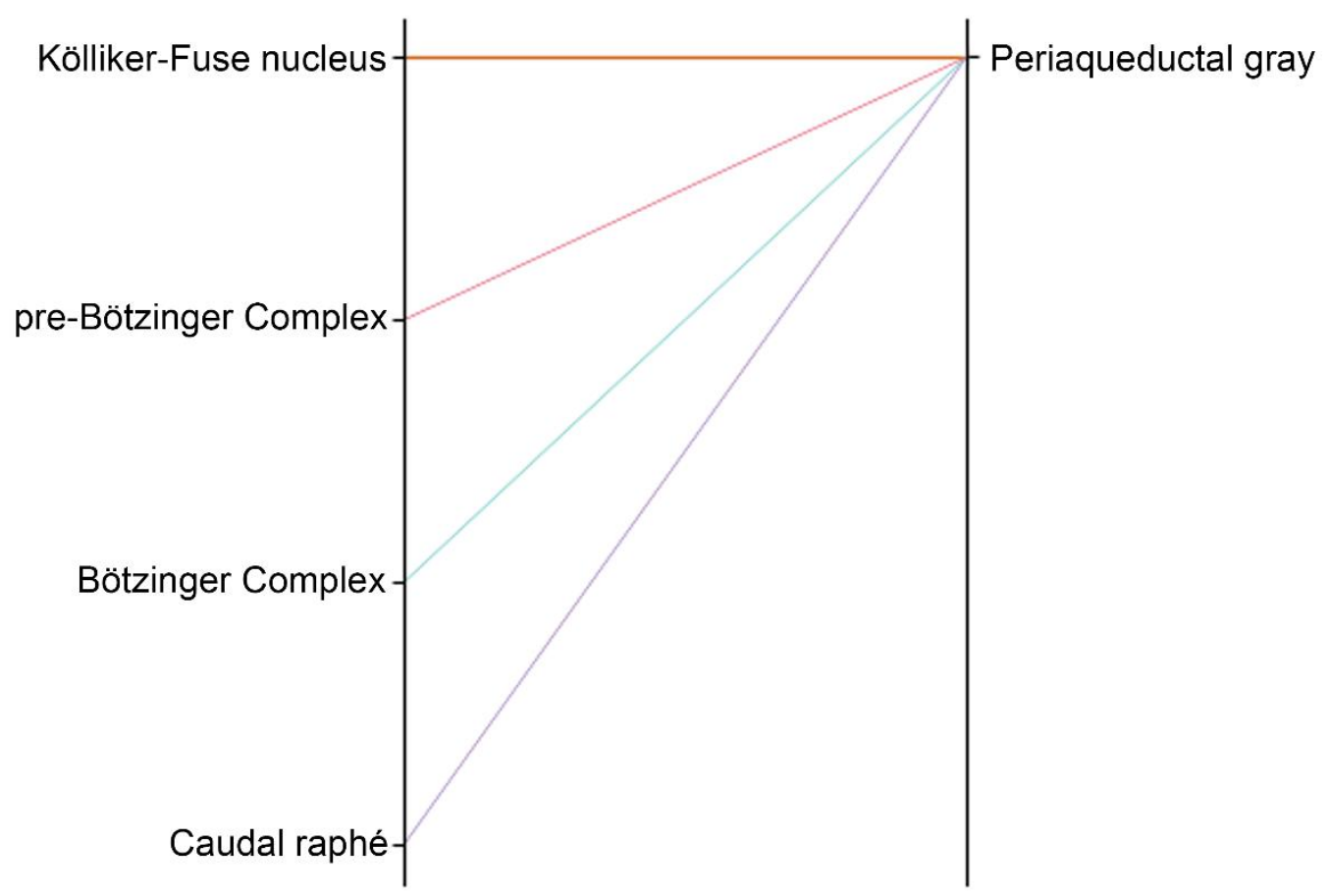

Figure 4. Summary of the relative strength of projections arising from the midbrain PAG columns (dmPAG, dIPAG, IPAG, and vIPAG; A), and the ponto-medullary regions (KFn, preBötC, BötC, and the caudal raphé; $B$ ) in a connectivity map. The weight of connecting lines are proportional to the normalized maximal number of CT-B-labeled neurons found in the specific source of descending projections. Abbreviation: PAG = periaqueductal gray. 